\title{
El espacio individual en la hiper-densidad
}

Buena parte de la actual configuración urbana de territorio de esta ex colonia inglesa y la presión de una sociedad entregada al neoliberalismo. La alta densidad poblacional obliga a usar los espacios de la ciudad como extensión natural de lo doméstico: una traslación posible gracias a la superposición de actividades en los estratos urbanos y a una red compleja y eficiente de transporte público. Palabras clave: Urbanismo - China, Hong-Kong, densidad poblacional, movilidad, transporte público.

Much of Hong Kong's urban configuration is the result of frictions generated by a society devoted to neoliberal economic bumping up against the limits imposed by the former British colony's miniscule territory. The high population density forces residents to use the city's spaces as a natural extension of their domestic space, a transference made possible by the superimposition of activities in the urban strata and a complex and efficient public transit network.

Key words: Urbanism - China, Hong Kong, population density, mobility, public transport. Hong-Kong es resultado de las fricciones entre el limitado
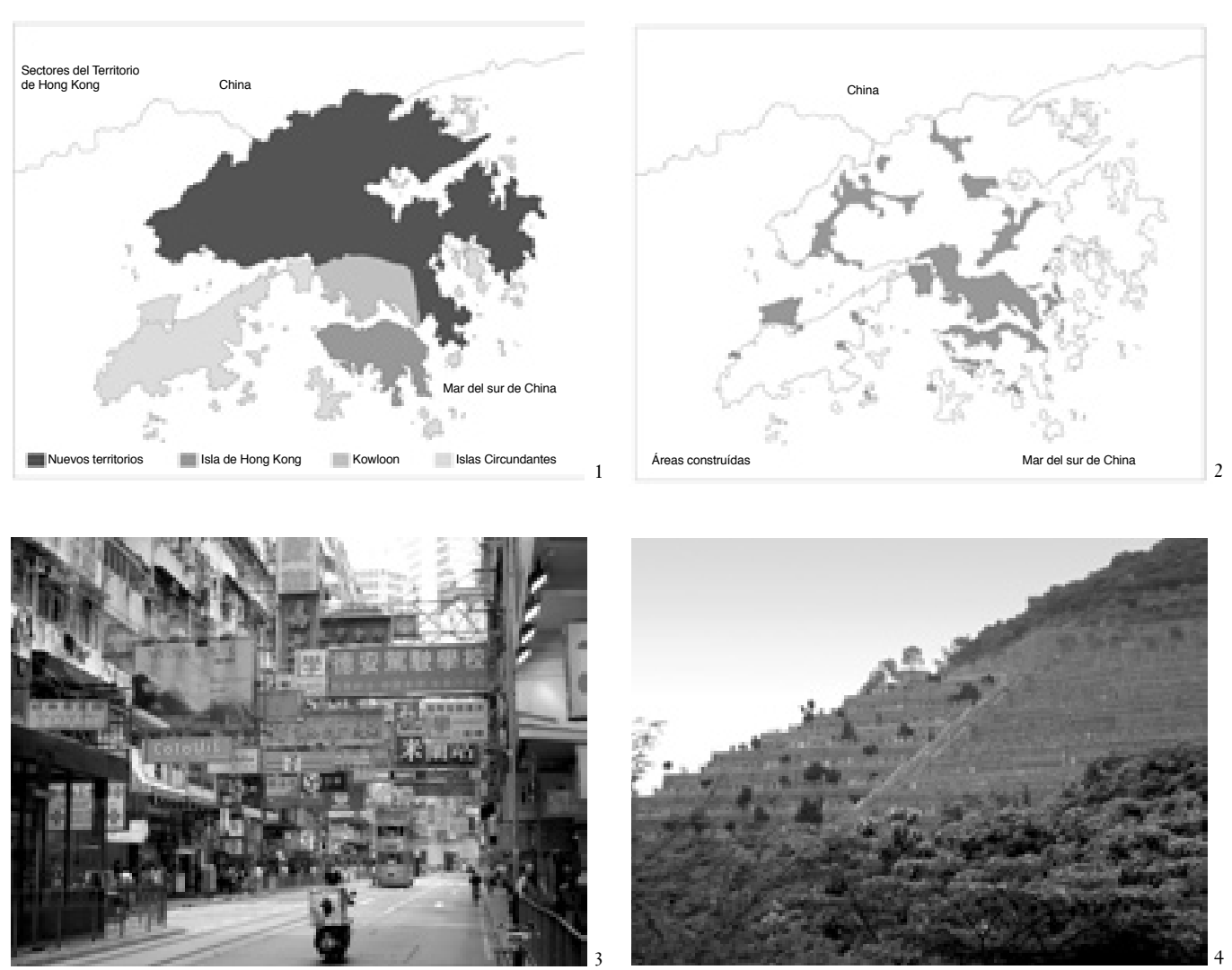

El limitado territorio de Hong Kong ${ }^{1}$ ha generado un gran número de experimentaciones con nuevas formas de vivir, trabajar, trasladarse y entretenerse, con acento particular en la densidad y la conectividad. Como arquitectos inmigrantes hemos querido explorar esta ciudad como parte de ella: buscar experiencias en los recorridos urbanos, entendiendo lo cotidiano no como lo banal y lo ordinario, sino como lo posible; mostrando la múltiple forma de esta ciudad y la diversidad de aproximaciones que un entorno tan complejo puede ofrecer.

\section{La ciudad}

Ciudad de excesos, dualidades y contrastes, Hong Kong sigue siendo hoy un lugar de encuentro entre oriente y occidente, un hábitat con una de las densidades poblacionales más altas del mundo. Hong Kong se reinventa y se transforma rápidamente; indudablemente su dinámica reside en la aceleración constante hacia calles más saturadas, edificios más altos y departamentos más chicos. Sin embargo, la ciudad que se redefine diariamente ha encontrado una continuidad en lo masivo, la velocidad y la proximidad.

Sus habitantes se mueven a la mayor velocidad posible entre centros comerciales, rascacielos y zonas dormitorio urbanas. La interacción con la ciudad parece ser ésa: demanda al hombre en su dimensión temporal, para apretarlo dentro de cubos cada vez más pequeños, hasta crear las mínimas unidades de transporte, vivienda, trabajo y ocio.

Paradojalmente, esta ciudad con apariencia caótica y múltiples perspectivas en el espacio público, contiene una completa uniformidad en sus espacios privados.

Hong Kong es el sistema resultante de un modo de vida hiper-densificado, que necesita de un fuerte soporte en el transporte masivo para optimizar el espacio de una ciudad-máquina.

\section{Vivienda - Nuevos territorios; 7:00 AM}

Despertar en una ciudad con una densidad de 6.250 babitantes por $\mathrm{km}^{2}$, en unidades residenciales que como celdas de un panal se suman unas a otras, multiplicando la monotonía, es parte del cotidiano de la gran masa bumana que babita Hong Kong.

En sólo $50 \mathrm{~m}^{2}$ de un bloque residencial que alcanza los 72 pisos de altura, un bombre de clase media de unos 40 años vive con su esposa, sus dos hijos, su madre y un sobrino (figs. 8 y 9).

La producción masiva de viviendas públicas $\mathrm{y}$ desarrollos residenciales privados ha resultado en una arquitectura que no trata el diseño del espacio, sino que es solamente la expresión superficial de una ilusión colectiva. Todo se reduce a buscar un desarrollo orientado a la movilidad; la población de Hong Kong antepone la localización y la proximidad sobre la espacialidad y la privacidad.

La urbanización desmedida tiene como consecuencia una evidente crisis del espacio individual. De la fórmula de repetir y multiplicar módulos habitacionales prediseñados a partir del estándar de lo mínimo, resultan estructuras que no contemplan situación ni contexto (ventanas bloqueadas, ventilación insuficiente, falta de privacidad $\mathrm{y}$ desorientación del habitante).

Se intenta a toda costa minimizar el espacio privado privilegiando el espacio público del movimiento, limitando lo propio sólo a lo justo. Este espacio mínimo, sumado al factor de la hiper-densidad urbana, origina la pérdida de la particularidad y la homogeneización de la arquitectura.

Los desarrollos residenciales masivos han eliminado tantas actividades del espacio de la vivienda, que el espacio público ha debido suplir las necesidades y carencias del espacio privado. Muchas actividades domésticas ahora pertenecen a lo urbano: restaurantes, gimnasios, cines, etc., llenan las calles para reemplazar el espacio individual perdido. La optimización del espacio implica entonces no sólo la verticalidad y el uso del territorio, 


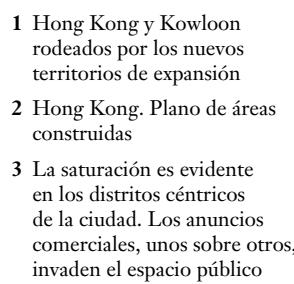

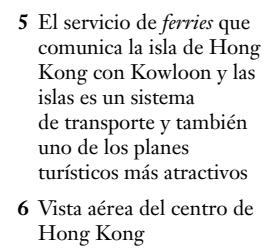

7 Multitud de peatones circulan por la ciudad

8 Desarrollo residencial.

Chai Wan

9 Uno de los tantos edificios Cesidenciales en el área de Chai Wan, una zona preferida por los residentes de Hon al centro de la ciudad
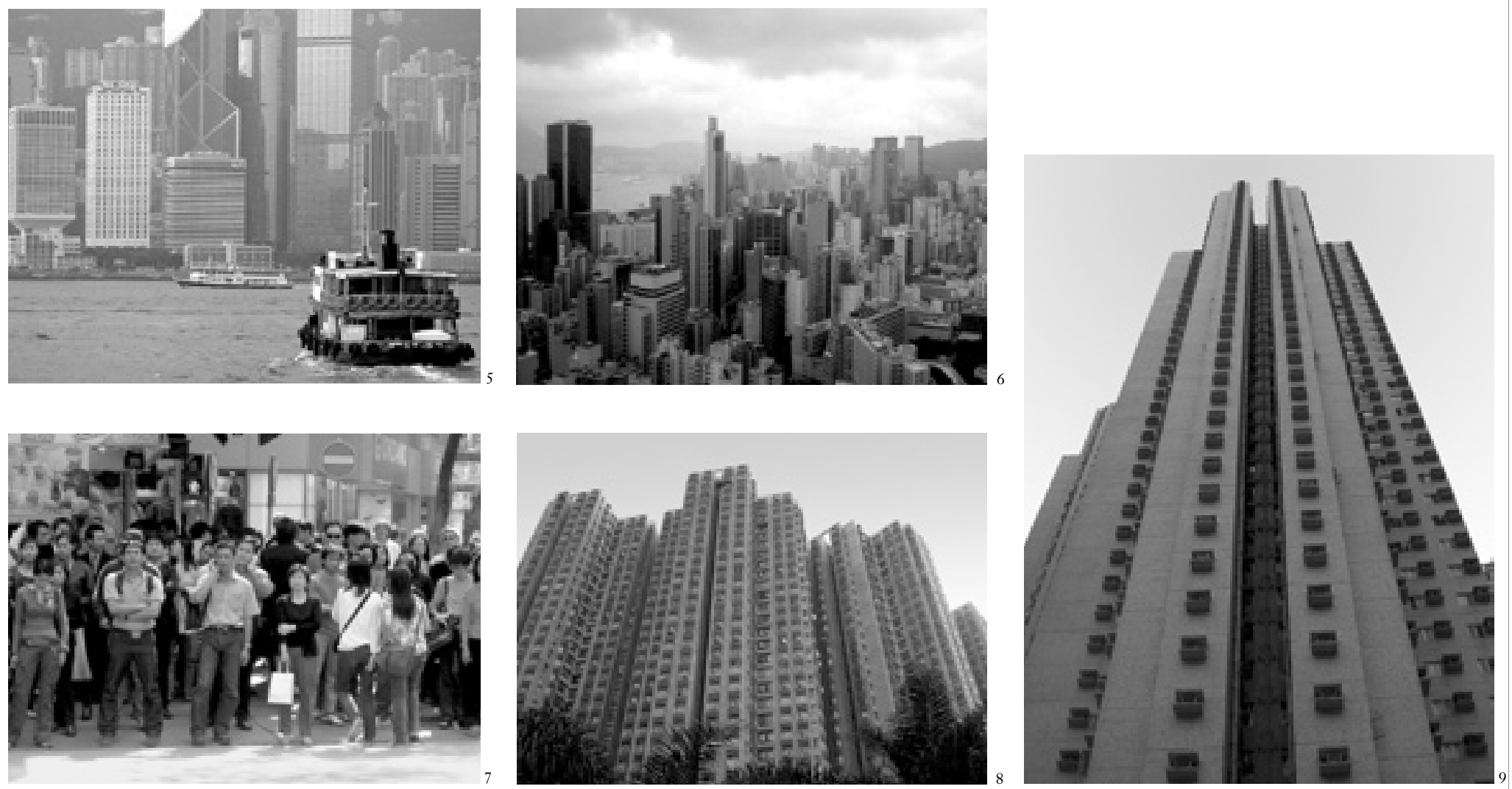

sino que además abarca todo un sistema que expande actividades y movimientos a cada nivel de infraestructura.

\section{Transporte - Kowloon; 8:00 AM}

Uno de los 11.015 .309 pasajeros que utilizan diariamente el transporte público camina por la estación de Mongkok para abordar uno de los trenes del MTR (Mass Transit Railway). Al momento de su llegada se cierran las puertas del vagón; a pesar que va con el tiempo justo el pasajero no se inmuta, pues sabe que el próximo tren tardará exactamente 1 minuto en llegar. MTR inicia sus operaciones antes de las 6:00 AM y termina pasada la 1:00 AM, con trenes de frecuencias entre 1 a 3 minutos, dependiendo de los horarios y necesidades. Se baja en la estación de Admiralty y toma uno de los 18.000 taxis que operan las 24 horas y que mueven por día 1,6 millones de pasajeros. El recorrido de esta mañana no ba incluido el uso de los modernos autobuses, ni de los cientos de ferries que comunican las islas y el continente.

El sistema de transporte masivo es el centro de gravedad de la ciudad: un sistema complejo que funciona prácticamente 24 horas al día, los 365 días del año. Hong Kong es en estado constante un flujo dinámico: una ciudad que se define más a través de flujos y movimientos que por sus estructuras permanentes. El sentido del espacio convencional se debilita frente al concepto de movilidad; los desplazamientos han asumido la organización del territorio, y el destino se ha convertido en el punto de paso. En Hong Kong el transporte masivo se encarga de unir los centros financieros de alta densidad ubicados en zonas céntricas de la ciudad con desarrollos residenciales hiperdensos que coexisten con la naturaleza. Es tal la importancia de la movilización que el valor de los distintos centros urbanos reside en su capacidad de conexión con otros puntos, más que en su relevancia histórica, social o geográfica. Para Hong Kong un nodo de transporte es sinónimo de urbanidad. Las redes se han convertido en una nueva forma de colonizar el espacio, por lo que la ciudad ha crecido en líneas, relacionando cada centro urbano a una estación de transporte.

\section{Oficina - Wan Chai; 9:00 AM}

En una de las 380 ferias anuales que se llevan a cabo en el centro de convenciones de Hong Kong, un hombre de negocios proveniente de Sudamérica camina entre los miles de asistentes, recorriendo los stands dedicados a presentar productos chinos al mercado occidental.

Luego de algunas conversaciones se dirige a una apretada oficina a pocas cuadras del lugar. En un edificio de 68 pisos de altura que alberga a más de 1.000 empresas de múltiples nacionalidades, cierra un negocio por 2 millones de dólares. Ha venido a Hong Kong por 4 días y volverá en 6 meses para la siguiente feria.

El desarrollo de centros empresariales y de negocios ha sido fundamental en la definición de la compleja red de movilidad de la ciudad. Las oficinas son sinónimo de eficiencia, densidad y conectividad, todo bajo el dinamismo del floreciente mercado industrial y financiero asiático.

Hong Kong ha tenido que desarrollar esquemas de soporte para recibir diariamente no sólo a los habitantes de la ciudad, sino además a miles de trabajadores que se trasladan desde la China Continental. Como centro financiero y empresarial, Hong Kong atrae una increíble población flotante que trabaja y se moviliza dentro del mismo sistema complejo, incrementando la necesidad por una organización espacial hipereficiente y limitada.

La optimización en el uso del territorio en la verticalidad busca expandir un alto número de servicios y actividades a todos los estratos urbanos. Los individuos organizados verticalmente y transportados masivamente en un edificio de oficinas son un ejemplo tangible de la magnitud y complejidad de las estructuras espaciales de la ciudad. 
$R=$ tesureser.

in - noves

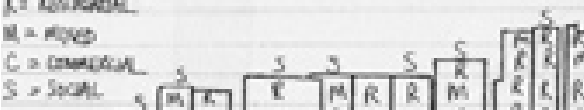
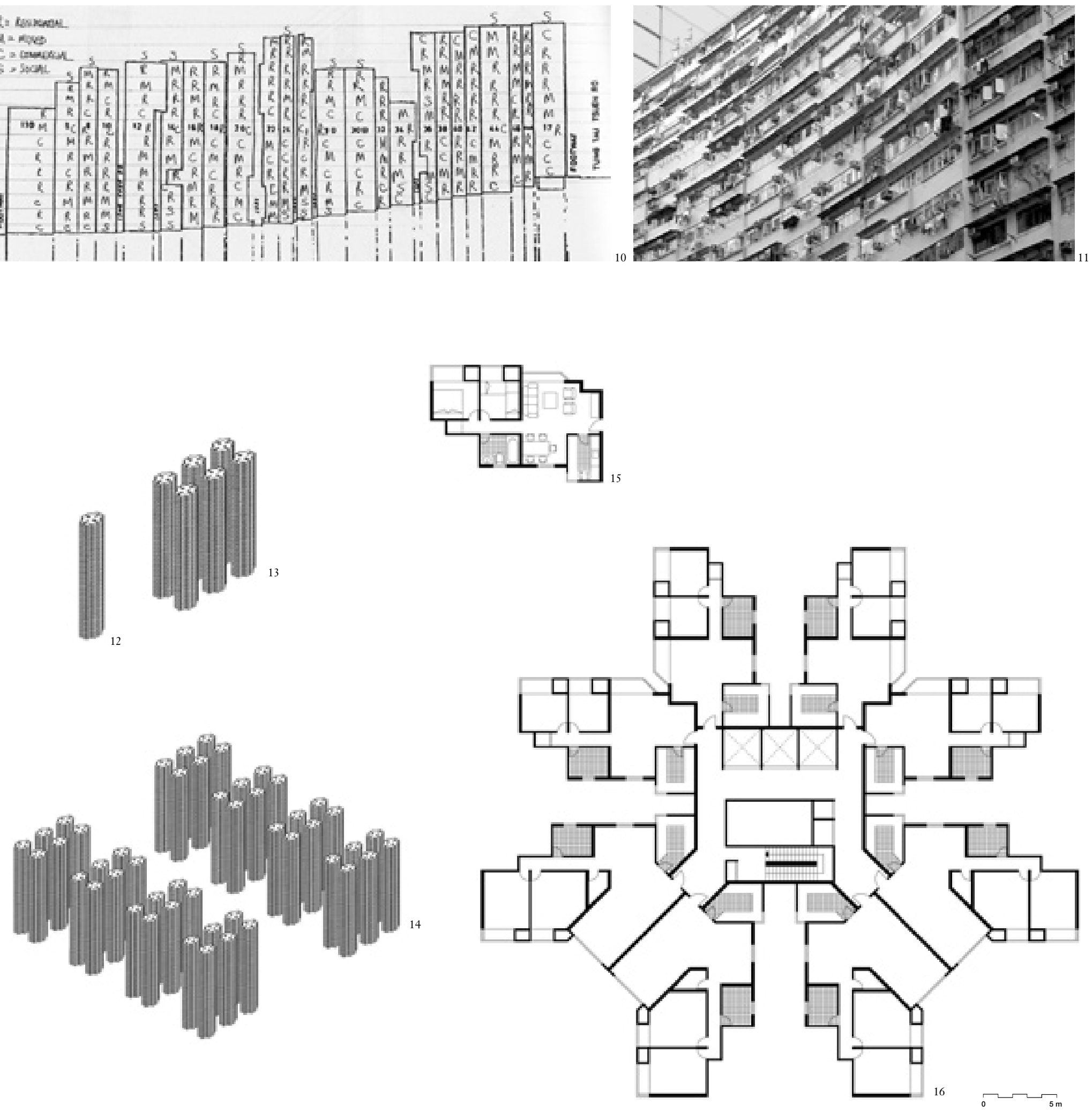

133.456 unidades: 2 ha / 16.000 habitantes / 1 colegio, 1 centro comercial, 1 cen recreativo, 1 centro comunitario, 1 área de
esparcimiento al aire libre, 1 línea de bus de

1 línea de bus de acercamiento al sistema de
transporte masivo, 1 sistem transporte masivo, 1 sistem de basura 1 centro comerial, 1 colegio,

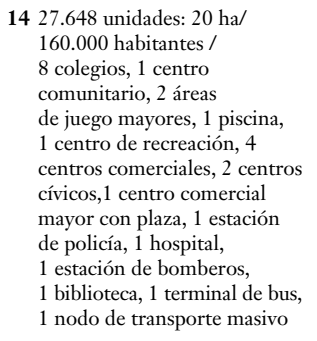

27.648 unidades: $20 \mathrm{~h}$ 60.000 habitantes /

8 colegios, 1 centro

de juego mayores, 1 piscina,

creación, 4

ciales, 2 cen

policía, 1 hospita

biblioteca, 1 terminal de bus

administración, casillas de

correo, estacionamiento

(1 cada 5 deptos.),

de basura, sala de juegos

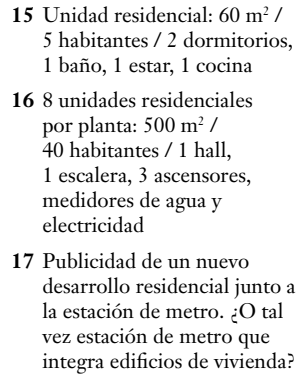

18 Vista del centro de convenciones y centro financiero de la ciudad

19 y 20 Pasarelas integradas a lo edificios. El acceso a las oficinas es inmediato

21 Midlevels escalator: La escalera mecánica más larga del mundo transportando ciudadanos a través de sectores residenciales, comerciales y de oficinas

$$
\begin{aligned}
& 22 \text { Hong Kong. } \\
& \text { Plano de líneas de metro } \\
& 23 \text { Hong Kong. } \\
& \text { Plano de vías principales } \\
& 24 \text { Hong Kong. } \\
& \text { Plano de rutas de ferry } \\
& \text { conectando sectores } \\
& \text { residenciales con estaciones } \\
& \text { de transporte } \\
& 25 \text { Sector residencial. Grupos } \\
& \text { y grupos de complejos } \\
& \text { habitacionales }
\end{aligned}
$$



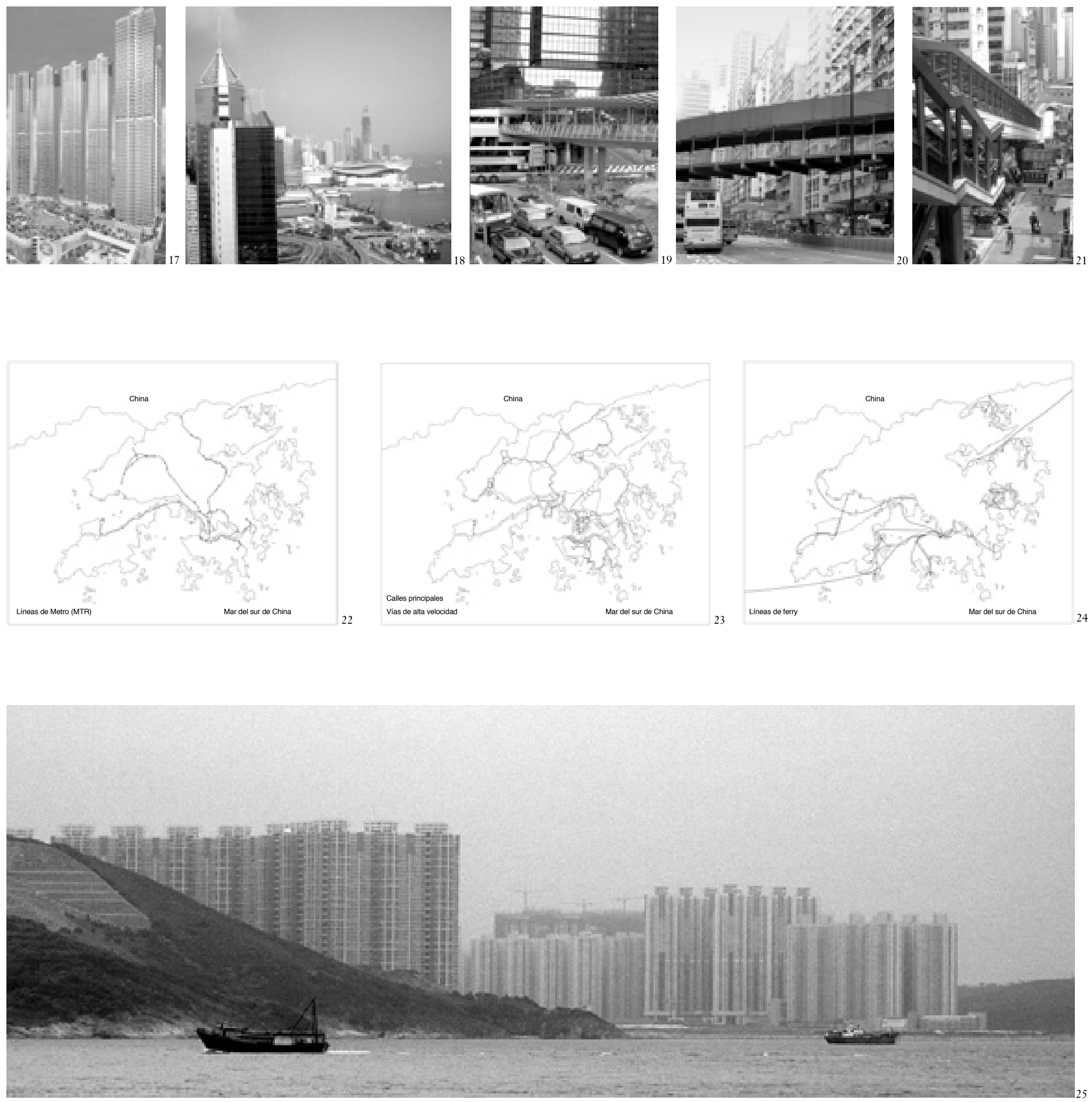

\section{Vías peatonales - Central, 1:00 PM}

Después de una mañana de trabajo una empleada de banco se dirige con otros compañeros a almorzar a un restaurante ubicado en el piso 6 de un edificio céntrico. El recorrido es constante y fluido: en sólo 8 minutos transitan sobre la ciudad a través de un laberinto de pasarelas y escaleras peatonales recorriendo una distancia mayor a $1 \mathrm{~km}$. No encuentran automóviles ni semáforos, sino accesos de edificios, restaurantes y comercio construidos en altura.

Encontrarse como peatón en medio de Hong Kong supone encontrarse entre masas, recorriendo fragmentos de ciudad y articulando una serie de percepciones discontinuas. El perfil de las calles se dibuja a través de un espacio compacto que dirige las multitudes en todas direcciones.

Pasarelas elevadas, interiores de edificios, centros comerciales, escaleras mecánicas y callejones congestionados son conectores que garantizan el flujo de la ciudad, recorriéndola en su interior y permitiendo al paseante ser testigo de la variedad de situaciones simultáneas en Hong Kong, produciendo una relación intermitente y compleja entre el espacio privado y el espacio público. Dominado por la movilidad, el espacio público deja espacios residuales mínimos que encuentran la promesa de la vida urbana a través del comercio.

Para entender una ciudad hipercongestionada como Hong Kong se hace necesario recomponer los fragmentos de un sistema caótico, a través de un concepto recurrente: la búsqueda del espacio individual. Cuando lo mínimo se convierte en la medida irreductible del espacio, la ciudad misma se convierte en una extensión de la vivienda. Finalmente es la agitación desbordante en la ciudad, su pulso acelerado, la vida escondida en cada rincón, lo que determina su carácter. ARQ 